\title{
Smoking Cessation by Electronic Cigarettes and Applied Artificial Intelligence
}

\author{
Henglong Ma1', Charlie Huang²
}

${ }^{1}$ Department of Electrical Engineering and Computer Science, Cleveland State University, Cleveland, $\mathrm{OH}$, USA

${ }^{2}$ School of Health and Rehabilitation Sciences, Ohio State University, $\mathrm{OH}$, USA

DOI: $10.47611 /$ jsr.v9i1.798

\section{ABSTRACT}

Numerous fields and administrations are embracing smart robotics. Despite the vast advancements in robotics for health care, there is limited practice and examination on utilizing electronic cigarettes (e-cigarettes, e-cigs, ENDS) and applied artificial intelligence (AI) to aid tobacco consumers to quit smoking. E-cigs and AI are certainly new tools that have the potential in helping Americans reduce smoking. It can be valuable for society to determine if e-smoking (smoking e-cigs) can become a healthier alternative than traditional smoking. This idea is worth studying more; according to Centers for Disease Control and Prevention, e-cigarettes have the potential to benefit some people but may harm others, scientists still have a lot to learn about whether e-cigarettes are effective in helping adults quit smoking. Additional input from researchers are needed to understand long-term health effects of e-cigarettes.

\section{Introduction}

Smoking may be the most preventable cause of death. The CDC estimates that the use of tobacco cigarettes is responsible for 480,000 deaths annually. Smoking also costs the United States billions of dollars each year -- $\$ 170$ billion in direct medical care for adults and more than $\$ 156$ billion in lost productivity due to premature death and exposure to secondhand smoke (Ross, 2016).

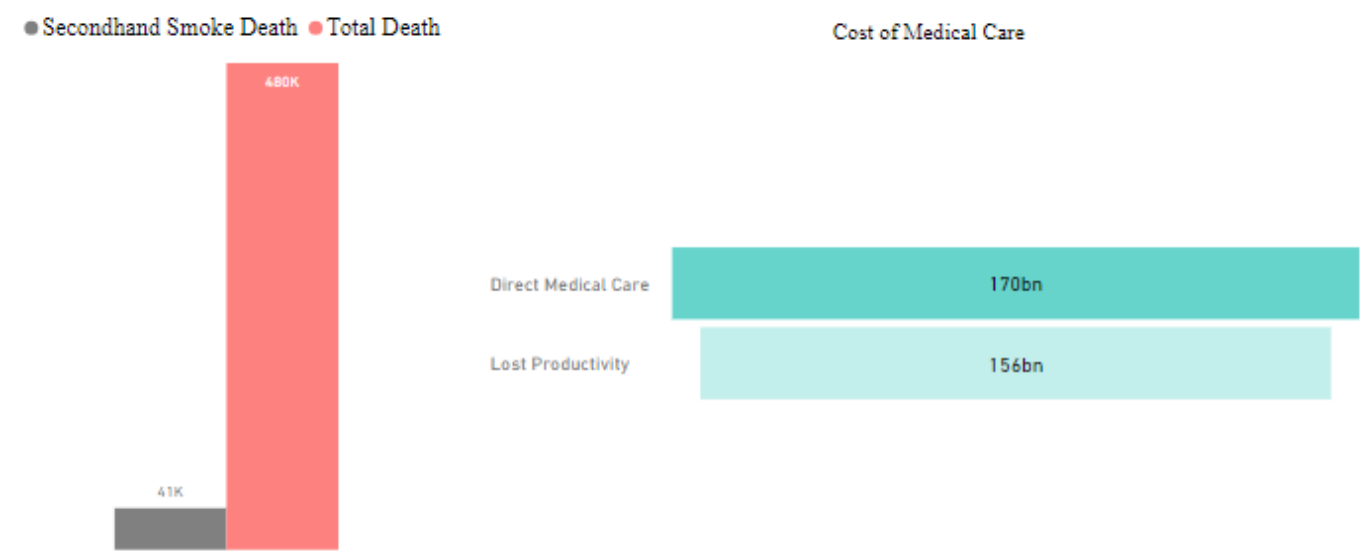

Figure 1: Death and Medical Care Cost by Smoking (Original data from Ross; model above made by Power BI) 
In the last ten years, new smoking cessation approaches have emerged such as the electronic cigarette (ecigarette or e-cig), which is a tobacco-free device powered by a battery to simulate the vapor effects of real smoking. The appearance of an e-cig differs based on the generation type. For example, first gen devices look identical to real tobacco cigarettes, but they are better than real tobacco cigarettes for health and environmental reasons. The first gen devices are designed to only work once or a few times and then must be disposed of. The second, third, and more newer gen devices are bulkier; they may look like thick cigars and pens and have greater robust features like larger rechargeable batteries so users can re-use the device more than a few times ("E-cigarettes: Facts, Stats and Regulations," n.d.). Future generations may give users superior enhancements not limited to AI voices, diagnostic systems, receptors, and personalized messages. These integrations are what Falkowska (2018) might call RAISA (short for robots, artificial intelligence, and service automation).

The health reason for using e-cigs rather than real tobacco cigarettes is because allegedly e-cigs do not make tar, carbon monoxide, hydrogen cyanide and other lethal elements which are found in cigarettes (Ross, 2016). According to the National Cancer Institute, tar is a chemical substance made when tobacco is burned, and it contains most of the cancer-causing and other harmful chemicals found in tobacco smoke. Therefore, the health and environmental costs of using e-cigarettes are lower than the costs of using tobacco cigarettes.

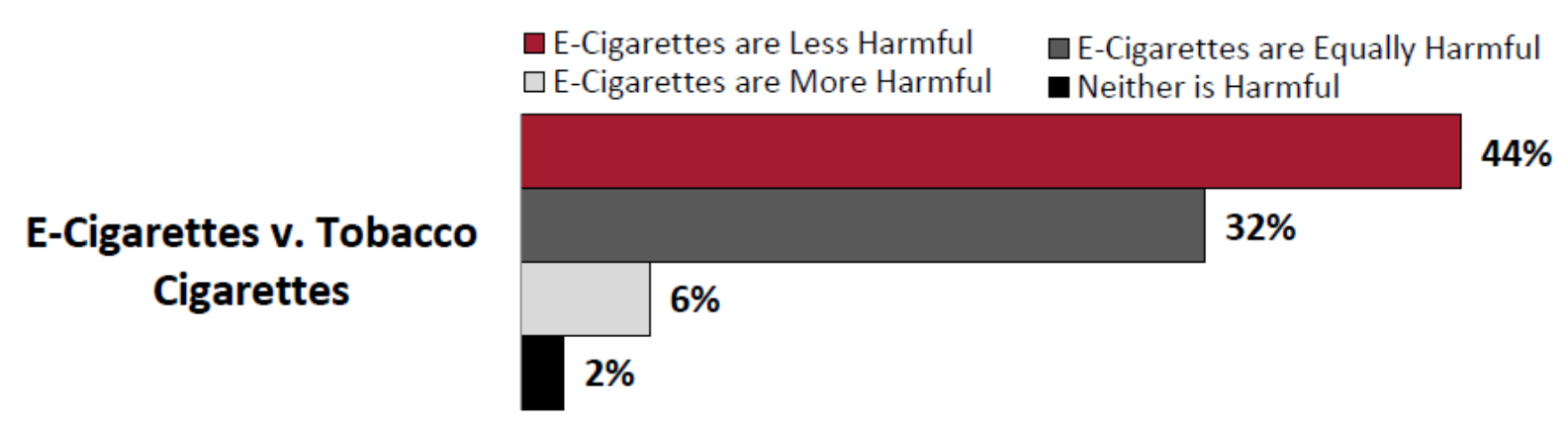

Figure 2: The perceived harm of e-cigarettes compared with tobacco cigarettes

Figure 2, Harvard's research data, demonstrates how adults feel about e-cigarettes compared with tobacco cigarettes. This data shows that the adoption of e-cigarettes is on the rise. It is important to inform adults of the lower risks of ecigs and find sound evidence on their effectiveness because only $44 \%$ of adults think they are less harmful than normal cigarettes. E-cigs can potentially help more people stay alive while also saving money and the environment.

\section{Purposes and Methods}

The primary purpose of this paper is to evaluate whether electronic cigarettes can be effective in promoting smoking cessation in U.S. adults. To select relevant literature, the following keywords were searched on the PubMed database: "smoking cessation", "e cigarettes", "adults", and "United States". Results were filtered to only include randomized controlled trials (RCTs), and they must be published in the last decade. The query returned 19 studies, of which 4 were included in this review. Main reason for excluding other studies was because of irrelevant results.

The secondary purpose of this paper is to critically review and hypothesize scenarios in which AI interventions could improve smoking cessation outcomes. To select relevant literature, the following keywords were searched on the PubMed database: "smoking cessation", "adults" and "artificial intelligence". 
Results were filtered to only include randomized controlled trials (RCTs), and they must be published in the last decade. The query returned 3 studies, all of which are included in this paper. Only 1 study of 3 is conducted in the United States.

\section{Results}

Lechner et al. (2015) conducted a cross-over randomized controlled trial in the Midwest region of the United States to assess the potential differences in the smoking cessation rates for use of first versus second gen e-cigs. The subjects consisted of twenty-two tobacco smokers, mostly Caucasian with a mean age of twenty-nine years old. Subjects smoked approximately fifteen cigarettes per day. Subjects were required to meet eleven requirements (not all listed here) to participate in the study; major requirements were being eighteen years or older, smoking at least five cigarettes each day, and having limited or no prior use of e-cig products. There were slightly more males than females who participated; a few requirements were responsible for excluding more females. Some subjects were randomized to receive first-gen products initially while other subjects received second-gen products. However, no subjects received both product types on the same day. Immediately before and after product use, the researchers asked participants to rate perceived aspects of their health on The Mood and Physical Symptoms Scale, which is made up of multiple five and six-point Likert scales. After analyzing the ratings, Lechner and researchers mainly found that second generation e-cigarettes appear to work better in decreasing nicotine withdrawal than first generation e-cigarettes.

Tseng et al. (2016) conducted a three-week, two-arm, double-blinded, randomized controlled trial on adult smokers in New York City (NYC) to assess the effect of electronic cigarettes on smoking reduction. The subjects consisted of ninety-nine people; about one-third were females and two-thirds were males. Participants, blacks and whites were both around thirty percent, whereas Hispanic and non-Hispanic were twenty-five and fifteen percent respectively. College education of participants shown in Figure 4. Major conditions for inclusion were that participants needed to be twenty-one years or older but less than thirty-five years, smoke at least ten cigarettes per day, be able to provide consent, and own a cell phone that could receive text messages. Major conditions for exclusion were previous use of e-cigs in the past two weeks, using alternative tobacco products, and receiving a severe drug use disorder score. Eighty of ninety-nine subjects completed all follow-up assessments up to week three. At the end of week three, the researchers measured that the number of cigarettes per day decreased significantly for the intervention group compared to the placebo group. Other measurements were determined by Smoking Behavioral Dependence Scale, which uses a 5-point Likert scale, and Readiness to Quit Ladder, which uses a 10-point Likert scale. In Tseng's paper the baseline characteristics were described and compared between two treatment groups using $\mathbf{t}$ tests and chi-square statistics analysis. At the end of three weeks of study, participants were able to reduce smoking significantly with the help of E-cigarettes as shown in Figure 3.

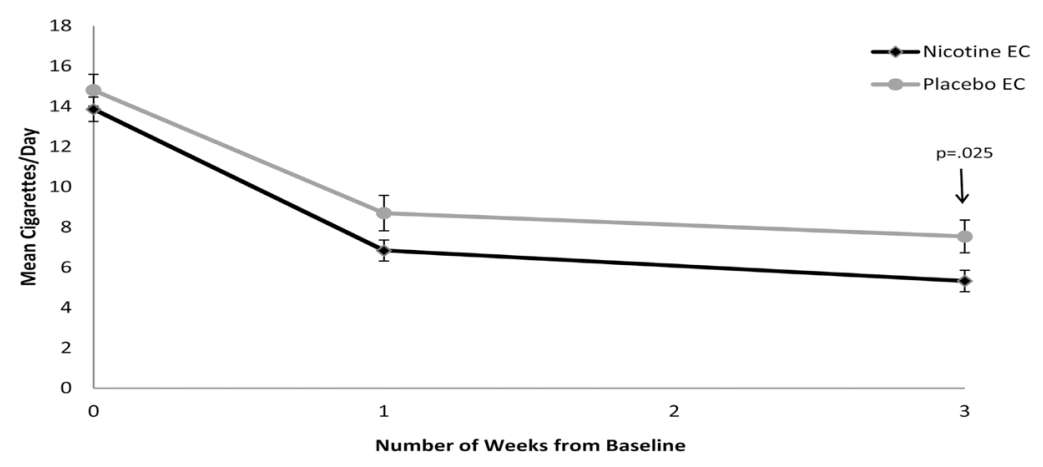

Figure 3. Time (in weeks) vs. changes in daily cigarettes smoked 


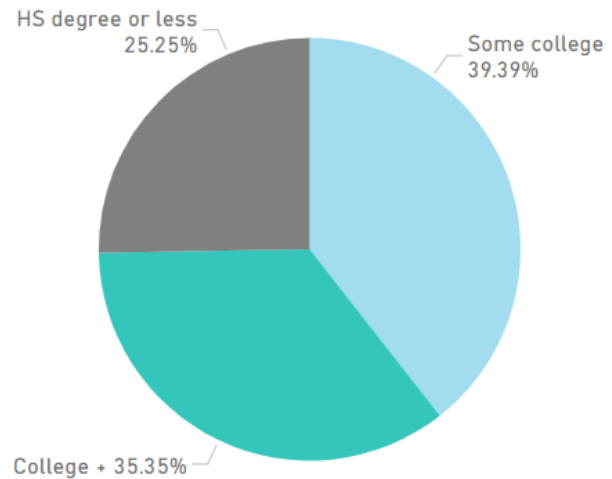

\begin{tabular}{lr} 
Education & \# of Participants \\
\hline Some college & 39 \\
College + & 35 \\
\hline HS degree or less & 25 \\
\hline Total & $\mathbf{9 9}$
\end{tabular}

Figure 4. Participants' Education Level (Original data from Tseng et al.; model above made by Power BI)

Litt, Duffy, and Oncken (2016) conducted a six week, two-factorial, randomized controlled trial in Greater Hartford, Connecticut, to assess how different flavors consumed with e-cigarettes may affect smoking rates. The subjects consisted of 88 males and 88 females who were randomly assigned to one of four e-cig groups. Each participant was required to complete daily logs of how much they used traditional cigarettes and e-cigarettes. Some participants in select e-cig groups were allowed refillable choices of the flavor they wanted to smoke. Inclusion and exclusion criteria were like previous studies; inclusion criteria consisted of smoking at least ten daily cigarettes, age of eighteen to fifty-five years, and ability to read and sign an English consent form. Over 300 study subjects called their offices to potentially register for the trial. Final participants had a mean age of 36 years, were seventy percent White, nineteen percent Black, eight percent Hispanic, and three percent Other; participants smoked an average of seventeen cigarettes per day. After six weeks, the researchers found that participants using menthol flavor for their e-cigs received the largest drop and the smallest drop in smoking cessation transpired among those who chose chocolate and cherry flavors. They also found that the normal cigarette smoking rates decreased more than fifty percent, from about sixteen to seven cigarettes per day.

Carpenter et al. (2017) conducted a three-week naturalistic and randomized controlled trial, to assess how likely adult smokers in the south-east parts of the United States would stop smoking after trying e-cigs or the electronic nicotine delivery systems (ENDS) as referred to by the researchers in this study. Subjects consisted of more females than males; the average participant's age was forty-two years old and sixty and forty percent were white and black persons respectively. Major requirements to be included in the study were eighteen years or older, smoke at least five cigarettes per day for at least one year and have not used ENDS before and actively use email. Results were collected from three randomized groups, two groups were exposed to different amounts of nicotine in their ENDS, and one group acted as the control group. Two flavors, menthol and tobacco, were offered to participants in the two intervention groups. The researchers found that people in the control group did not reduce smoking whereas people in the two intervention groups significantly reduced smoking. It should be noted that subjects were provided ENDS free of charge as well as other reimbursements.

Moving on to artificial intelligence results, only one of the three randomly controlled trials is conducted in the United States. As such, only the results of this one study will be presented in this section; the other two studies will appear in the discussion section. Sadasivam, Borglund, Adams, Marlin, and Houston (2016) conducted 30 days research, compared a randomized standard CTHC experiment (CTHC is short for computer-tailored health communication) to a novel machine learning system known as CTHC: Patient Experience Recommender System for Persuasive Communication Tailoring (PERSPeCT). They leveraged an innovative hybrid machine learning recommender system PERSPeCT that selects and sends motivational messages to previous participants (846 of 900 participants, explicit feedback). 120 smokers that were aged 18 years or older, English speakers, and with Internet access were eligible to 
participate. Smokers were randomized to receive either PERSPeCT or standard CTHC tailored messages at ratio of 2:1 by daily email. Major reasons for unequal random allocation: increased experience with the experimental CTHC; it allowed for more subgroup evaluation since the standard system was effective and PERSPeCT was very new.

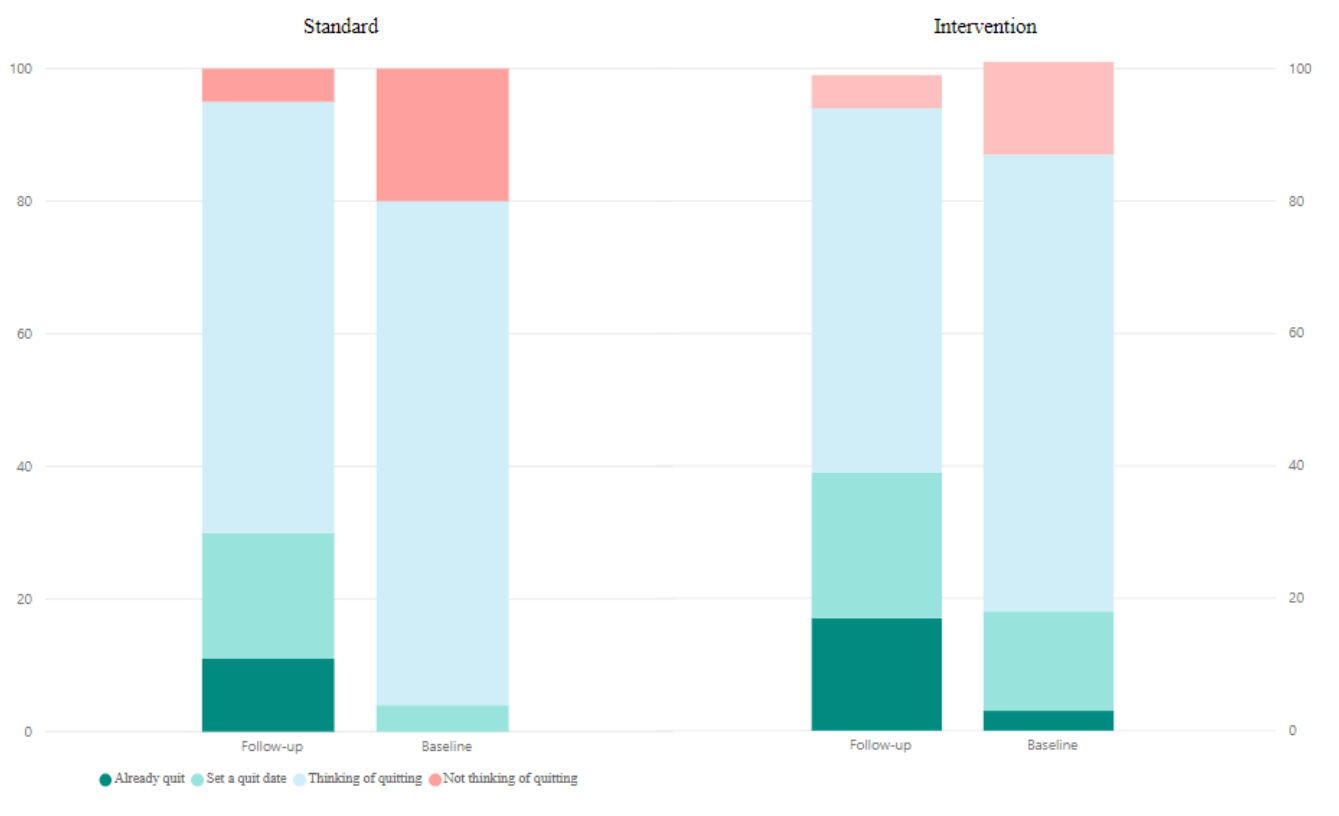

Figure 5. Baseline and follow-up readiness to quit status in standard vs. intervention -- percentage stacked bar chart. Higher blue-green bars on the right (vs. left) correlate with increased smoking cessation (Original data from Sadasivam et al.; model above made by Power BI)

\section{Discussion and Limitations}

Overall, these studies show that some e-cigs tend to have more effectiveness in promoting smoking cessation than placebo or no treatment. Three studies (Litt et al., 2016; Carpenter et al., 2017; Tseng et al., 2016) asked participants to use e-cigarettes for at least three weeks. Measurements were different for all articles, though most consisted of Likert scales. First generation e-cigarette BluCig is utilized by Lechner et al. (2015) research; the researchers also studied the second-generation e-cigarette JoyeTech E-go C. A major weakness for the study by Lechner et al. (2015) was the small sample size, which limits generalizability. A couple notable limitations of the Tseng study and the Lechner study: most participants were young adults with some college education (average age of 28 or 29) — young people may be more willing to adopt to new technology. Results of both studies are slightly biased since they treat more male participants. Only one specific e-cig brand NJOY King Bold and tobacco flavor had been tested in the Tseng study, and it is unknown how many flavors were tested in the Lechner study. Further study with different e-cig devices (by brands) and flavors (including chemical safety) is needed to comprehend the true effectiveness of Nicotine e-cigarette compared to the Placebo e-cigarette.

A main strength for Litt et al. (2016) would be they used large and equal gender sample sizes; however, racial diversity may be a biased point in their study. A great strength of the study by Tseng et al. (2016) is its strong study design through a double-blinded RCT, which is different from previous research articles; however, a weakness is that the researchers only tested participants with one specific e-cig brand and flavor. Carpenter et al. (2017) does extremely well regarding the fact its participants are almost equal in the gender and race category. One of the weak points of their study design is that they provided the intervention for free and most of their participants were forty years old, 
which is older than most participants of other studies. A major limitation for the results of all studies is that they are short-term findings. Long-term trials should be conducted in the future.

A comparable study design to Carpenter is Sadasivam et al. (2016), who pay their participants a small reward after completing each part of their study. They inform us in their "previous study" that a few basic messages (standard CTHC) sent by AI or computer(s) sent to smoking participants are significantly better than no messages (control group). In their newly designed CTHC system, PERSPeCT, they once again produce results that show that specialized messages such as benefits of quitting (motivational reasoning) and spending time on something important or new (behavior training) are more useful, efficient and persuasive than basic or generic messages in helping participants make the final call to quit smoking. Although their sample size is large, they do suggest that their participants are frequent visitors to or near their University hospital.

Furthermore, Meyer et al. (2016) discusses something identical to CTHC; they call it "computer-based tailoring of counseling letters". The study is presumed to gather participants living in Germany. Their results exemplify that some intervention for smoking reduction is better than no intervention (control group is $8.5 \%$ ). One of their two interventions (12.9\%) is slightly less successful than the other (14.7\%).

Additional automated (AI powered CTHC) systems like "QuitCoach" have been utilized in Australia by Balmford, Borland, Benda, and Howard (2013), who recruited Australian residents who have personal mobile phone numbers and were not diagnosed with serious mental disorders. The "QuitCoach" system, created in 2003, has sent tailored messages to more than 30,000 smokers. They say the main takeaways of their study is that there is a great deal of interest in automated interventions and multiple interventions are more popular than single interventions. These three studies confirm that the results of AI and "personalized messages" appear more effective than sending common and possibly non-interactive and one-time information to tobacco consumers. The AI and standard message designs can be analogous to the term RAISA (see introduction for definition).

\section{Conclusion}

After examining the evidence presented in the above articles, e-cigarettes tend to be useful in helping American smokers quit. Certain e-cig brands, model types and flavors such as menthol may be more effective than others in promoting smoking cessation. However, the FDA has not approved e-cigarettes as a way to quit smoking, and more research is needed on to establish whether e-cigarettes would be effective for an American to quit smoking and to better understand the health effects of e-cigarettes. Like alcohol content, most e-cigarettes contain nicotine in different amounts. In order for adult smokers to achieve any meaningful health benefits from e-cigarettes, they would need to switch to e-cigarette brands that contain or release lower amounts of nicotine over time. Also, adult smokers should reduce other forms of smoking with eventual goal that they can stop smoking cigarettes and other tobacco products completely. If possible, the final goal would be to discontinue reliance of e-cigarettes completely to achieve the maximum health benefit. In the earlier months of the year 2020, the FDA has banned fruit and mint flavors vaping cartridges. This limit might have been good or bad. There has not been much research on e-cigarette flavors. If e-cigarettes can be proven to be safe and beneficial, it would be important to educate users on how to safely use e-cigs; one safe practice may be keeping the device and e-liquid - the flavored substance poured into e-cigarettes - away from children (Ross, 2016). Additionally, it would also be important to inform users how to properly recycle and dispose of e-cigarettes, including the batteries. The results of the studies searched can only be generalized to American adult smokers who smoke daily, usually five or more cigarettes. Most studies are likely to have used different types and brands of e-cigs and e-liquid. Previous studies that claim e-cigs are not effective in smoking cessation only experiment with first-gen e-cigs (Lechner et al., 2015). Future research should focus on comparing second, third gen and newer devices as well as different brands and flavors to look for greater differences and potential for smoking cessation. According to Oppenheim (2019), in other nations like England, there is compelling evidence showing that e-cigs are less harmful than smoking and there are few AI and smart apps that can help people stop smoking or practice safe smoking. For example, Pivot is an app that can show important data values such as carbon monoxide levels. Similarly, there are likely very few 
apps that will monitor e-cigarette use, although in the case of e-cigs, it may be unknown what chemicals should be paid attention to.

The use of e-cigarettes, particularly fourth, fifth, and future generations, should invest in machine learning systems to maximize the possibility of smoking reduction and cessation. E-cigarettes may or may not be effective for smoking cessation; everything has its pros and cons. For instance, recently JUUL is under a lot controversy because of potential health risks to individuals using it. The American Lung Association has concerns about health risks of ecigarettes. Since nicotine is mixed with propylene glycol, formaldehyde and acrolein, these have the potential to cause lung damage. Inhaling these might create toxicity. Though e-cigarettes may help a current smoker, neither the CDC nor the FDA has claimed that e-cigarettes are safe. Additionally, there are risks of second-hand e-cigarette emissions as well. They include diacetyl, benzene, nickel, tin and lead which are linked to lung disease. This article is not meant to promote e-cigarettes, but rather exhibit some initial findings on a multiple of different approaches on smoking cessation. Long term and in-depth randomized controlled trials, reviews, and analyses about e-cigarettes will help the world paint a constructive picture of e-cig usage.

\section{Acknowledgements}

We thank Dr. Crystal Dunlevy, our research advisor, for her generous support.

\section{References}

Balmford, J., Borland, R., Benda, P., \& Howard, S. (2013). Factors associated with use of automated smoking cessation interventions: Findings from the eQuit study. Health Education Research, 28(2), 288-299. https://doi.org/10.1093/her/cys104

Carpenter, M. J., Heckman, B. W., Wahlquist, A. E., Wagener, T. L., Goniewicz, M. L., Gray, K. M., ... Cummings, K. M. (2017). A naturalistic, randomized pilot trial of e-cigarettes: Uptake, exposure, and behavioral effects. Cancer Epidemiology Biomarkers \& Prevention, 26(12), 1795-1803. https://doi.org/10.1158/10559965.EPI-17-0460

E-cigarettes: Facts, stats and regulations. (n.d.). Retrieved April 8, 2019, from Truth Initiative website: https://truthinitiative.org/research-resources/emerging-tobacco-products/e-cigarettes-facts-stats-and-regulations

Falkowska, E. (2018). Retrieved April 8, 2019, from DigitalMeBU website: https://digitalmebu.wordpress.com/2018/06/15/raisa-on-the-public-sector-express/

Lechner, W. V., Meier, E., Wiener, J. L., Grant, D. M., Gilmore, J., Judah, M. R., ... Wagener, T. L. (2015). The comparative efficacy of first- versus second-generation electronic cigarettes in reducing symptoms of nicotine withdrawal: Comparative efficacy of electronic cigarettes. Addiction, 110(5), 862-867. https://doi.org/10.1111/add.12870

Litt, M. D., Duffy, V., \& Oncken, C. (2016). Cigarette smoking and electronic cigarette vaping patterns as a function of e-cigarette flavourings. Tobacco Control, 25(Suppl 2), ii67-ii72. https://doi.org/10.1136/tobaccocontrol-2016-053223

Meyer, C., Ulbricht, S., Haug, S., Broda, A., Bischof, G., Rumpf, H.-J., \& John, U. (2016). Motivating smokers to quit using computer-generated letters that target either reduction or cessation: A population-based randomized controlled trial among smokers who do not intend to quit. Drug and Alcohol Dependence, 166, 177186. https://doi.org/10.1016/j.drugalcdep.2016.07.009

Oppenheim, S. (n.d.). Can tech change the way we tackle smoking? Retrieved April 8, 2019, from Forbes website: https:/www.forbes.com/sites/serenaoppenheim/2019/01/29/can-tech-change-the-way-we-tackle-smoking/

Ross, J. (2016, July 25). E-cigarettes: Good news, bad news. Retrieved April 8, 2019, from Harvard Health Blog 
website: https://www.health.harvard.edu/blog/electronic-cigarettes-good-news-bad-news-2016072510010

Sadasivam, R. S., Borglund, E. M., Adams, R., Marlin, B. M., \& Houston, T. K. (2016). Impact of a collective intelligence tailored messaging system on smoking cessation: The perspect randomized experiment. Journal of Medical Internet Research, 18(11), e285. https://doi.org/10.2196/jmir.6465

Tseng, T.-Y., Ostroff, J. S., Campo, A., Gerard, M., Kirchner, T., Rotrosen, J., \& Shelley, D. (2016). A randomized trial comparing the effect of nicotine versus placebo electronic cigarettes on smoking reduction among young adult smokers. Nicotine \& Tobacco Research, 18(10), 1937-1943. https://doi.org/10.1093/ntr/ntw017 